\title{
First-principles study of bulk and surface oxygen vacancies in $\mathrm{SrTiO}_{3}$ crystal
}

\author{
V.E. Alexandrov ${ }^{1, a}$, E.A. Kotomin ${ }^{1,2}$, J. Maier ${ }^{1}$, and R.A. Evarestov ${ }^{3}$ \\ 1 Max-Planck-Institut für Festkörperforschung, Heisenbergstrasse 1, 70569, Stuttgart, Germany \\ 2 Institute of Solid State Physics, University of Latvia, Riga 1063, Latvia \\ 3 Department of Quantum Chemistry, St.Petersburg State University, 26 Universitetskiy Prospekt, Stary Peterhof \\ 198504, Russia
}

Received 20 March 2009 / Received in final form 23 July 2009

Published online 10 October 2009 - (c) EDP Sciences, Società Italiana di Fisica, Springer-Verlag 2009

\begin{abstract}
The structural and electronic properties of the neutral and positively charged oxygen vacancies ( $F$ and $F^{+}$centres) in the bulk and on the (001) surfaces of $\mathrm{SrTiO}_{3}$ crystal are examined within the hybrid Hartree-Fock and density functional theory (HF-DFT) method based upon the linear combination of atomic orbital (LCAO) approach. A comparison of the formation energy for surface and bulk defects indicates a perceptible propensity for the segregation of neutral and charged vacancies to both $\mathrm{SrO}$ and $\mathrm{TiO}_{2}$ surface terminations with a preference in the latter case which is important for interpretation of space charge effects at ceramic interfaces. It is found that the vacancies reveal more shallow energy levels in the band gap on surfaces rather than in the bulk, in particular, on the $\mathrm{TiO}_{2}$ surface. The charged $F^{+}$ centre has significantly deeper energy levels both in bulk and on the surfaces, as compared with the neutral $F$ centre.
\end{abstract}

PACS. 61.72.jd Vacancies - 71.15.Ap Basis sets and related methodology - 61.72.jn Color centers

\section{Introduction}

$\mathrm{ABO}_{3}$ perovskite-type oxides comprise a broad family of crystalline materials with a rich spectrum of technologically appealing properties. $\mathrm{SrTiO}_{3}$ crystal deserves particular attention as a representative model for the wide class of large bandgap perovskites. At room temperature and under ambient conditions, $\mathrm{SrTiO}_{3}$ adopts a cubic structure which undergoes a phase transition to a tetragonal phase below 105 K [1]. Doped oxide materials with the aptitude to tolerate oxygen deficiencies have numerous functional applications such as gas sensors, solid oxide fuel cells, permeation membranes, electrochemical and memory devices $[2,3]$. Oxygen vacancies, being very important structure elements in real (often nonstoichiometric) perovskites, play a prominent role in a number of phenomena. Deliberate variation of oxygen content allows one to modify $\mathrm{SrTiO}_{3}$ not only from an $n$-type conductor (at low oxygen partial pressure) to $p$-type (at high pressure) or even a superconducting state, but also from an electronic to an ionic conductor. Oxygen vacancies are also believed to be a crucial factor in trapping electrons causing the highly undesirable leakage current in microelectronic de-

\footnotetext{
${ }^{a}$ e-mail: v.alexandrov@fkf.mpg.de
}

vices such as, e.g., the dynamic random access memory capacitors [4].

Recent first-principles calculations have provided very useful information about the atomic and electronic structure, chemical bonding and covalency effects for nondefective bulk [5-7] and surfaces of $\mathrm{SrTiO}_{3}$ [8-11]. It has been shown, e.g., that the (001) surfaces ( $\mathrm{SrO}-$ and $\mathrm{TiO}_{2}-$ terminated) are preferable over the polar (011) surfaces (Sr-, O- and TiO-terminated) [11], whereas the $\mathrm{TiO}_{2}$ facet is very close in formation energy to the $\mathrm{SrO}$ surface [10]. It has been also revealed that there is a considerable increase in the $\mathrm{Ti}-\mathrm{O}$ chemical bond covalency near surfaces what should affect the properties of surface defects and adsorption phenomena.

Compared with bulk defects, the properties of oxygen vacancies at surfaces have received little attention so far. The properties of neutral oxygen vacancy with two trapped electrons known as a $F$ centre is relatively well understood owing to a series of mostly DFT studies [12-18]. Despite the recent DFT modelling of the oxygen-deficient $\mathrm{TiO}_{2}$-terminated surface [15], which predicted vacancy segregation to the surface with an $1.5 \mathrm{eV}$ energy gain, the understanding of the surface $F$ centre is still scarce. Thus, it is important to compare the formation energies of the $F$ centre on both $\mathrm{SrO}$ and $\mathrm{TiO}_{2}$ surfaces, in order to 
find out which termination facilitates the segregation process most. Understanding of defect segregation is a key for analysis of space charge effects at ceramic interfaces including grain boundaries. Another important question of the $F$ centre energy level position in the band gap has not been addressed yet due to the well-known systematic underestimate of the band gap within the DFT method and thus misplacing the $F$ centre level relative to the conduction band. Thirdly, the properties of charged oxygen vacancies and its impact on the functionality of semiconductor devices have recently attracted increasing attention [14,19-21]. However, the electronic properties of the vacancy with a single electron $\left(F^{+}\right.$centre) in $\mathrm{SrTiO}_{3}$ and their relation to those of neutral vacancy have not been considered so far.

In this paper, we study both $F$ and $F^{+}$centres in the bulk and on the $\mathrm{SrO}$ - and $\mathrm{TiO}_{2}$-terminated $\mathrm{SrTiO}_{3}(001)$ surfaces. We mainly focus on a comparison of the electronic properties of surface and bulk vacancies, including the defect level position, and the vacancy segregation energy from the bulk to the surfaces.

\section{Computational details}

The simulations were carried out in the framework of the HF-DFT hybrid approach with the B3PW exchangecorrelation functional [22] using the LCAO-based CRYSTAL06 computer package [23]. This approach provides very good agreement between theory and experiment for many fundamental properties including the band gaps of perovskites [6]. Since the use of a proper basis set is an essential ingredient in the LCAO calculations, we used a basis set previously optimized for $\mathrm{SrTiO}_{3}[6]$. In order to analyze properties of the oxygen vacancy, we employed a "ghost" wave function which means that the basis set of the removed oxygen atom is retained at the vacant site. For the calculation of a free oxygen atom as a reference in computing vacancy formation energy, we used an atomic 6-311 basis set. The Monkhorst-Pack [24] mesh of k-points was adopted for the summation over the Brillouin zone. The $2 \times 2 \times 2 \mathrm{k}$-points sampling for the bulk and the $3 \times 3$ mesh was used for surface calculations. The chosen computational scheme allowed us to obtain the lattice constant $\mathrm{a}_{0}=3.892 \AA$ for $\mathrm{SrTiO}_{3}$ crystal which is in perfect agreement with the experimental value extrapolated to $\mathrm{O} \mathrm{K}$ $(3.89 \AA[25])$, as well as the band gap of $3.6 \mathrm{eV}$ vs. $3.3 \mathrm{eV}$ in experiment. The latter point is very important for the correct placing of one-electron energy levels of the oxygen vacancies within the band gap.

In defect modelling, we exploited a periodic supercell approach [17]. For the bulk calculations, we considered two different supercells with 80 and 160 atoms where the latter, as alluded to below, is large enough to substantially reduce the spurious interactions between defects in neighboring cells. According to our simulations, the ground state of the neutral $F$ centre is singlet, while the energy of triplet state for the relaxed 80-atom supercell is $0.12 \mathrm{eV}$ higher. Modelling of the charged $F^{+}$centre were

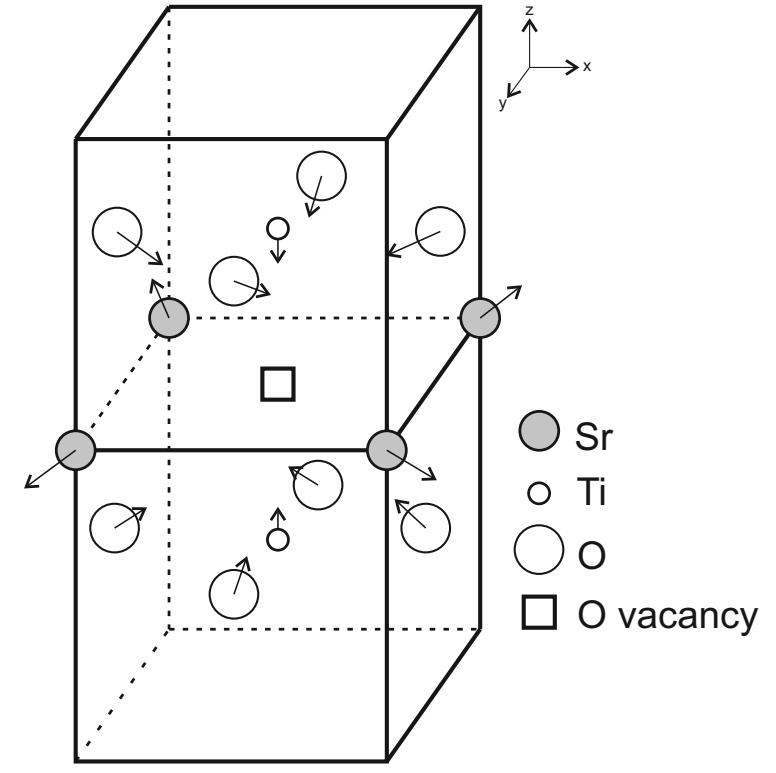

Fig. 1. Displacements of the vacancy-nearest atoms (three closest atomic shells) due to the lattice distortion in $\mathrm{SrTiO}_{3}$ crystal with the neutral $F$ centre.

performed maintaining the neutrality of a cell by a replacing $\mathrm{Sr}^{2+}$ ion by a singly charged $\mathrm{Rb}^{+}$ion close in size. To eliminate the effect of such artificial charge compensator on the calculated $F^{+}$properties, we placed the $\mathrm{Rb}^{+}$ion as far as $6.15 \AA$ away from the vacancy and found that further moving it away changes the total energy by less than $0.01 \mathrm{eV}$.

To model the surface $F$ centre, we treated $\mathrm{SrO}$ and $\mathrm{TiO}_{2}$-terminated symmetrical slabs consisting of 7 stacking $\mathrm{SrO}$ and $\mathrm{TiO}_{2}$ planes and a $3 \times 3$ surface supercell with an oxygen vacancy on opposite sides. This allowed us to benefit from the higher symmetry due to the presence of a central mirror plane, while the treatment of an asymmetrical 6-layer slab (SrO terminated on one side and $\mathrm{TiO}_{2}$ terminated on another one) with a proper cation stoichiometry did not significantly affect our results. In order to explore the charged $F^{+}$centre on the surfaces and to avoid non-negligible interactions between defects on opposite slab sides, we used a 6-layer slab with only one vacancy on either $\mathrm{SrO}$ or $\mathrm{TiO}_{2}$ surface and a compensating $\mathrm{Rb}^{+}$ion within the slab. Note that we used a cubic lattice constant in all structure optimization calculations, only lowering the point symmetry to the tetragonal.

\section{Results and discussion}

After performing a complete atomic structure optimization, we determined the displacements of atoms around the two types of oxygen vacancies (Tab. 1) and the directions of atomic displacements are schematically shown in Figure 1. It is seen that for the neutral $F$ centre atomic displacements are very small and become even smaller with decreasing oxygen vacancy concentration, thus accounting 
Table 1. The displacements of atoms (in \% of the lattice constant a $a_{0}$ ) nearest to the neutral bulk oxygen vacancy $(F$ centre) for supercells with $6.25 \%$ (80-atom) and $3.125 \%$ (160-atom) defect concentration (atomic displacements around the $F^{+}$are given in parentheses). $N$ is the number of symmetrically equivalent atoms in each atomic shell around the vacancy (symmetry decrease for $F^{+}$leads to the shell splitting). Positive sign means displacement outward from a vacancy.

\begin{tabular}{cccccccc}
\hline & Unrelaxed & \multicolumn{9}{c}{} & \multicolumn{5}{c}{ Radial displacements } \\
Atom & distance & \multicolumn{2}{c}{ Unrelaxed coordinates } & $N$ & 80 -atom & 160-atom \\
\hline $\mathrm{Ti}$ & $\mathrm{a}_{0} / 2$ & 0 & 0 & $\pm \mathrm{a}_{0} / 2$ & 2 & -1.21 & $-0.96(2.40)$ \\
$\mathrm{O}$ & $\mathrm{a}_{0} / \sqrt{2}$ & $\pm \mathrm{a}_{0} / 2$ & 0 & $\pm \mathrm{a}_{0} / 2$ & 8 & -1.14 & $-1.05(-1.59 ;-1.57)$ \\
& & 0 & $\pm \mathrm{a}_{0} / 2$ & $\pm \mathrm{a}_{0} / 2$ & & & \\
$\mathrm{Sr}$ & $\mathrm{a}_{0} / \sqrt{2}$ & $\pm \mathrm{a}_{0} / 2$ & $\pm \mathrm{a}_{0} / 2$ & 0 & 4 & 0.92 & $0.25(0.39 ; 0.43 ; 0.52)$ \\
$\mathrm{O}$ & $\mathrm{a}_{0}$ & $\pm \mathrm{a}_{0}$ & 0 & 0 & 4 & 0.45 & $0.37(0.30 ; 0.49)$ \\
& & 0 & $\pm \mathrm{a}_{0}$ & 0 & & & \\
$\mathrm{O}$ & $\mathrm{a}_{0}$ & 0 & 0 & $\pm \mathrm{a}_{0}$ & 2 & 0.40 & $0.58(-0.36)$ \\
\hline
\end{tabular}

Table 2. Calculated defect energy level $\Delta \varepsilon_{d b}^{\Gamma}(\mathrm{eV})$ with respect to the bottom of conduction band at the $\Gamma$ point of the Brillouin zone, its dispersion $\delta \varepsilon_{d b}(\mathrm{eV})$, the Mulliken charge $q(e)$ of the vacancy and the formation energies $E_{\text {form }}(\mathrm{eV})$ of the bulk $F$ and $F^{+}$centres relative to atomic oxygen (see explanation in the text), $d_{F-F}$ denotes the distance between periodically repeated defects $(\AA)$.

\begin{tabular}{ccccccc}
\hline Supercell & $d_{F-F}$ & Relaxation & $\Delta \varepsilon_{d b}^{\Gamma}$ & $\delta \varepsilon_{d b}$ & $q$ & $E_{\text {form }}$ \\
\hline 80-atom $(F)$ & 11.01 & unrelaxed & 0.84 & 0.13 & 1.28 & 9.17 \\
& & relaxed & 0.79 & 0.13 & 1.26 & 9.07 \\
160-atom $(F)$ & 13.48 & unrelaxed & 0.75 & 0.06 & 1.28 & 9.17 \\
& & relaxed & 0.77 & 0.06 & 1.26 & 9.06 \\
160-atom $\left(F^{+}\right)$ & 13.48 & relaxed & 1.20 & 0.05 & 0.82 & 8.41 \\
\hline
\end{tabular}

for the obtained small relaxation energies $(\sim 0.1 \mathrm{eV})$. It should be pointed out that our result is in contrast to that obtained within the pure DFT plane-wave calculations [17] where the displacements of the nearest Ti atoms were considerably larger and in the opposite direction (outwards from a vacancy). This discrepancy is not surprising in the light of the different electronic structure of the $F$ centre predicted by the two methods: the hybrid method predicts the classical neutral $F$ centre with two electrons well localized inside the vacancy, whereas pure DFT tends to largely delocalize the electron density, thus leading in fact to a positively charged vacancy, which obviously has a repulsive interaction with two surrounding Ti cations. The relaxation around the $F^{+}$centre differs considerably from that of the $F$ centre (Tab. 1) and the nearest Ti atoms displace outwards.

The band structure calculations show that the oneelectron energy levels of the occupied bulk $F$ and $F^{+}$defect bands lie below the bottom of conduction band (see Tab. 2 and band structure of the $F$ centre in Fig. 2). It is seen that the dispersions of these defect bands $\left(\delta \varepsilon_{d b}\right)$ throughout the Brillouin zone are particularly small for 160-atom cell, demonstrating a weak interaction between oxygen vacancies in neighboring supercells. One can recognize a noticeable difference in the defect level position which is much deeper for the $F^{+}$centre, being in line with the Green function method predictions for $\mathrm{SrTiO}_{3}[12]$ and first-principles calculation results obtained for other tran-

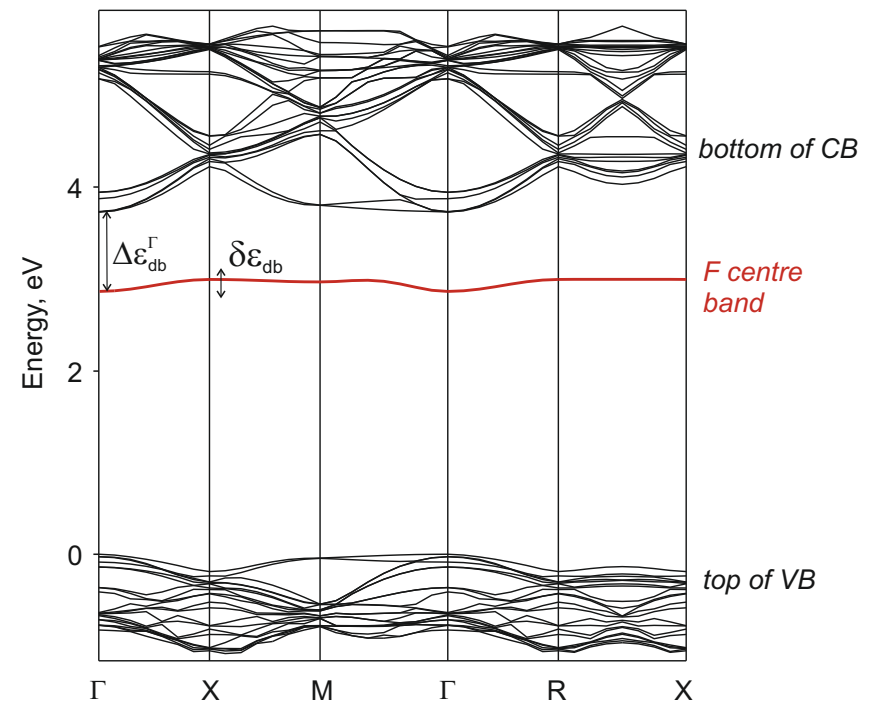

Fig. 2. (Color online) Band structure of the relaxed $\mathrm{SrTiO}_{3}$ crystal with a neutral $F$ centre in 80 -atom supercell. The top of valence band (VB) composed of $\mathrm{O} 2 p$ states and the bottom of conduction band (CB) composed of Ti $3 d$ states are shown. The vacancy band position with respect to the $\mathrm{CB}$ bottom $\left(\Delta \varepsilon_{d b}^{\Gamma}\right)$ and its dispersion $\left(\delta \varepsilon_{d b}\right)$ are listed in Tables 2 (bulk) and 4 (surface).

sition metal oxides [20]. The Mulliken population analysis indicates that an electron charge of about $\sim 1.3 \mathrm{e}$ is localized at the vacancy site while the residual $0.7 e$ of the missing $\mathrm{O}^{2-}$ ion is equally distributed among the two Ti ions nearest to the neutral $F$ centre. Note that the difference between the two defect one-electron levels is consistent with that calculated using the total energies of defect states for the Franck-Condon (vertical) excitations [20].

Defect formation energies are computed as follows:

$$
E_{\text {form }}\left(V_{O}\right)=E\left(\mathrm{SrTiO}_{3}: \mathrm{V}_{\mathrm{O}}\right)+E_{O}-E\left(\mathrm{SrTiO}_{3}\right)
$$

where $E\left(\mathrm{SrTiO}_{3}: \mathrm{V}_{\mathrm{O}}\right)$ and $E\left(\mathrm{SrTiO}_{3}\right)$ are energies of supercells with and without the vacancy, respectively, $E_{O}$ is the energy of a free oxygen atom in its ground (triplet) state. The obtained values are summarized in Table 2 for the bulk and Table 4 for the surface vacancies. Regarding 
Table 3. Displacements of atoms nearest to the surface $F$ centre ( $\%$ of $\left.\mathrm{a}_{0}\right): N$ is the number of symmetrically equivalent atoms in each shell around the vacancy and $M$ is the modulus of the displacements.

\begin{tabular}{llllllllllll}
\hline & \multicolumn{3}{c}{ SrO termination } & \multicolumn{6}{c}{$\mathrm{TiO}_{2}$ termination } \\
Atom & $N$ & $\Delta x$ & $\Delta y$ & $\Delta z$ & $M$ & Atom & $N$ & $\Delta x$ & $\Delta y$ & $\Delta z$ & $M$ \\
\hline $\mathrm{Ti}$ & 1 & 0.00 & 0.00 & 0.74 & 0.74 & $\mathrm{Ti}$ & 2 & -0.52 & -1.92 & -3.24 & 3.80 \\
$\mathrm{Sr}$ & 4 & 0.58 & 0.58 & -4.72 & 4.76 & $\mathrm{O}$ & 2 & 2.50 & 2.18 & -0.28 & 3.33 \\
$\mathrm{O}$ & 4 & -0.17 & 0.00 & 2.70 & 2.71 & $\mathrm{Sr}$ & 1 & 0.00 & -0.03 & 0.62 & 0.62 \\
$\mathrm{O}$ & 4 & 0.00 & 1.87 & 1.12 & 2.18 & $\mathrm{O}$ & 2 & 1.06 & 0.34 & -0.45 & 1.20 \\
$\mathrm{O}$ & 1 & 0.00 & 0.00 & -0.28 & 0.28 & $\mathrm{Sr}$ & 1 & 0.00 & -0.22 & 0.74 & 0.77 \\
$\mathrm{Ti}$ & 4 & 0.00 & 0.24 & 1.75 & 1.77 & $\mathrm{O}$ & 2 & 1.18 & 1.21 & -0.47 & 1.75 \\
\hline
\end{tabular}

Table 4. The properties of surface vacancies (see Tab. 2 for explanation of notation) obtained with an interdefect separation of $11.7 \AA$ for fully relaxed structures.

\begin{tabular}{lllll}
\hline Defect & Surface & $\Delta \varepsilon_{d b}^{\Gamma}$ & $\delta \varepsilon_{d b}$ & $E_{\text {form }}$ \\
\hline$F$ centre & $\mathrm{SrO}$ & 0.27 & 0.02 & 8.04 \\
& $\mathrm{TiO}_{2}$ & very shallow & - & 7.62 \\
$F^{+}$centre & $\mathrm{SrO}$ & 1.19 & 0.02 & 6.16 \\
& $\mathrm{TiO}_{2}$ & 0.81 & 0.002 & 4.87 \\
\hline
\end{tabular}

the defect formation energies, we should point out that this quantity for a charged state is a function of the electron chemical potential $\mu_{e}[26]$. It has been shown for the bulk case [14] that the creation of singly-charged oxygen vacancy is energetically more favorable than the neutral one for almost the entire range of admissible $\mu_{e}$. The stability of these two states alters only at high Fermi energy corresponding to the heavily $n$-type material.

Turning to the discussion of surface defects, we can see on the example of the neutral $F$ centre that the atomic displacements exhibit a more complex pattern because of both defect incorporation and surface relaxation (Tab. 3). This results in a larger perturbation area which spreads over several coordination spheres. The calculated formation energies of the surface vacancies (Tab. 4) are considerably smaller than in bulk. Such a strong propensity of the oxygen vacancies for segregation from the bulk to the surfaces is consistent with previous DFT predictions obtained for a $\sum=3(111)[10 \overline{1}]$ grain boundary in $\mathrm{SrTiO}_{3}$ [27], as well as for other perovskites [28].

Regarding the electronic properties, the surface $F$ centre turns out to be a much more shallow defect than that in the bulk, however, this depends largely on the surface termination. Thus, for the $\mathrm{TiO}_{2}$ surface, the defect level of the $F$ centre nearly degenerate the conduction band bottom and the surface becomes practically metallic. This is accompanied by a considerable electron density delocalization from the vacancy over the nearest ions.

The surface $F^{+}$centre is a deeper defect than the neutral vacancy, similar to the situation in the bulk. Due to the known high covalency of $\mathrm{Ti}-\mathrm{O}$ chemical bond especially at the surface, the electron density of the charged vacancy is well spread over the nearest Ti atoms resulting in a mixed character of the defect band level. These results are fairly consistent with the conclusions drawn from ultraviolet-photoemission spectroscopy measurements on reduced $\mathrm{SrTiO}_{3}$ (001) surfaces indicating the coexistence of both quite deep $(1.2 \mathrm{eV}$ below the bottom of the CB [29]) and shallow [30] levels due to surface oxygen vacancies.

Summing up, a comparative analysis of the atomic, electronic and energetic properties of the $F$ and $F^{+}$centres in $\mathrm{SrTiO}_{3}$ shows that the charged oxygen vacancy accommodates the peculiarities of having a stronger local lattice relaxation, a deeper one-electron energy level and considerably stronger propensity for the surface segregation. A common feature of both types of vacancies is the more shallow energy levels when being on the surfaces, particularly on $\mathrm{TiO}_{2}$ termination; however, the effect is less pronounced for the charged oxygen vacancy.

Authors are greatly indebted to G. Pacchioni, A. Shluger, Yu. Zhukovskii, S. Piskunov and R. Merkle for many fruitful discussions. This study was partly supported by FP7 MATERA project (EK).

\section{References}

1. P.A. Fleury, J.F. Scott, J.M. Worlock, Phys. Rev. Lett. 21, 16 (1968)

2. P.A. Cox, Transition Metal Oxides (Clarendon Press, Oxford, UK, 1995)

3. The Chemical Physics of Solid Surfaces - Oxide Surfaces, edited by P. Woodruff (Elsevier, Amsterdam, 2001)

4. S.H. Paek, E.S. Lee, S.H. Kim, J.Y. Seong, J.P. Mah, C.S. Park, J.S. Choi, J.H. Jung, Journal of Materials Science 33, 1239 (1998)

5. W. Zhong, R.D. Kingsmith, D. Vanderbilt, Phys. Rev. Lett. 72, 3618 (1994)

6. S. Piskunov, E. Heifets, R.I. Eglitis, G. Borstel, Comput. Mater. Sci. 29, 165 (2004)

7. Y. Xie, H.T. Yu, G.X. Zhang, H.G. Fu, J. Phys. Condens, Matter 20, 215215 (2008)

8. E. Heifets, R.I. Eglitis, E.A. Kotomin, J. Maier, G. Borstel, Phys. Rev. B 64, 235417 (2001)

9. D.S. Deak, Materials Science and Technology 23, 127 (2007)

10. S. Piskunov, E.A. Kotomin, E. Heifets, J. Maier, R.I Eglitis, G. Borstel, Surf. Sci. 575, 75 (2005)

11. E. Heifets, W.A. Goddard, E.A. Kotomin, R.I. Eglitis, G. Borstel, Phys. Rev. B 69, 035408 (2004) 
12. S.A. Prosandeyev, A.V. Fisenko, A.I. Riabchinski, I.A. Osipenko, I.P. Raevski, N. Safontseva, J. Phys. Condens. Matter 8, 6705 (1996)

13. R. Astala, P.D. Bristowe, Modelling and Simulation in Materials Science and Engineering 9, 415 (2001)

14. J.P. Buban, H. Iddir, S. Ogüt, Phys. Rev. B 69, 180102 (2004)

15. J. Carrasco, F. Illas, N. Lopez, E.A. Kotomin, Y.F. Zhukovskii, R.A. Evarestov, Y.A. Mastrikov, S. Piskunov, J. Maier, Phys. Rev. B 73, 064106 (2006)

16. D. Ricci, G. Bano, G. Pacchioni, F. Illas, Phys. Rev. B 68, 224105 (2003)

17. R.A. Evarestov, E.A. Kotomin, Y.F. Zhukovskii, Int. J. Quant. Chem. 106, $2173(2006)$

18. Y.F. Zhukovskii, E.A. Kotomin, R.A. Evarestov, D.E. Ellis, Int. J. Quant. Chem. 107, 2956 (2007)

19. P.E. Blöchl, Phys. Rev. B 62, 6158 (2000)

20. J.L. Gavartin, D.M. Ramo, A.L. Shluger, G. Bersuker, B.H. Lee, Appl. Phys. Lett. 89, 082908 (2006)
21. D.B. Strukov, G.S. Snider, D.R. Stewart, R.S. Williams, Nature 453, 80 (2008)

22. A.D. Becke, J. Chem. Phys. 98, 5648 (1993)

23. R. Dovesi, C. Saunders, V.R. Roetti, R. Orlando, C.M. Zicovich-Wilson, F. Pascale, B. Civalerri, K. Doll, N.M. Harrison, I.J. Bush, P. D'Arco et al., CRYSTAL-2006 User's Manual (University of Torino, Torino, 2006)

24. H.J. Monkhorst, J.D. Pack, Phys. Rev. B 13, 5188 (1976)

25. Ferroelectrics and Related Substances, New Series, Landolt-Bornstein (Springer Verlag, Berlin, 1969), Vol. 3

26. S.B. Zhang, J. Phys. Condens. Matter 14, R881 (2002)

27. R. Astala, P.D. Bristowe, J. Phys. Condens. Matter 14, 6455 (2002)

28. L.X. He, D. Vanderbilt, Phys. Rev. B 68, 134103 (2003)

29. R. Courths, Physica Status Solidi B-Basic Research 100, $135(1980)$

30. V.E. Henrich, G. Dresselhaus, H.J. Zeiger, Phys. Rev. B 17, 4908 (1978) 